\title{
Angiomixoma Pélvico Asociado a Endometriosis Infiltrativa Profunda. Reporte de un caso
}

\author{
Pelvic Angiomyxoma Associated to Deep Infiltrative Endometriosis. Case report
}

\author{
Carlos Manterola ${ }^{1,2}$; Ricardo San Martín ${ }^{2}$ \& Alfredo Chodowiecki²
}

\begin{abstract}
MANTEROLA, C.; SAN MARTÍN, R. \& CHODOWIECKI, A. Angiomixoma pélvico asociado a endometriosis infiltrativa profunda. Reporte de un caso. Int. J. Morphol., 37(2):677-681, 2019.

RESUMEN: El angiomixoma de la pelvis es un tumor mesenquimal inusual; que dado su alto potencial de recidiva es relevante que la exéresis quirúrgica sea lo más radical posible. Por su parte, la endometriosis infiltrativa profunda, es otra entidad poco frecuente que requiere de tratamientos complejos. La co-existencia de ambas patologías representa una situación absolutamente infrecuente; de los que hay muy pocos casos reportado en la literatura occidental. Se presenta el caso de una mujer de 41 años de edad que desarrolló ambas entidades nosológicas de forma concomitante y que fue tratada quirúrgicamente con buenos resultados.
\end{abstract}

PALABRAS CLAVE: Angiomixoma; angiomixoma pélvico; neoplasia mesenquimatosas.

\section{INTRODUCCIÓN}

El angiomixoma es una neoplasia de origen mesenquimático, de comportamiento agresivo, de aparición más frecuente en mujeres en edad reproductiva, premenopáusicas (Steeper \& Rosai, 1983; Linares Espinos et al., 2014). Es una lesión muy infrecuente, con alrededor de 300 casos descritos en la literatura (Adwan et al., 2004; Idrees et al., 2006; Haldar et al., 2010; Artigas Raventós et al., 2016). Son tumores de celularidad escasa, de estroma muy vascularizado y aspecto mixoide. Destaca la ausencia de atipias y escas actividad mitótica, con preeminencia de células fusiformes y estrelladas. Pueden expresar receptores para estrógenos y progesterona, lo que se asocia con efectos hormonales en su crecimiento (Rotmensch et al., 1993; Idrees et al.; Andres \& Mishra, 2007; Chen et al., 2017).

La mayoría de los casos son asintomáticos o presentar síntomas inespecíficos, por lo que el diagnóstico suele ser incidental (Choi et al., 2015). Si bien suelen comportarse como lesiones de comportamiento benigno, siendo poco frecuente el desarrollo de metástasis a distancia, tiene una elevada recurrencia local (Artigas Raventós et al.). El método de elección para seguimiento postoperatorio es la RNM, por medio de la cual, se pueden apreciar lesiones iso o hipointensa en comparación con el músculo en T1; y de alta intensidad de señal en imágenes en T2 (Llauger et al., 1998; Giraudmaillet et al., 2015; Chen et al.).
Las recidivas locales son frecuentes, con tasas reportadas entre $25 \%$ y $47 \%$; el $85 \%$ de las cuales se presenta en los primeros 5 años del postoperatorio (Sutton \& Laudadio, 2012; Kiran et al., 2013). El tratamiento es la exéresis quirúrgica completa, tanto en las lesiones primarias como en las eventuales recidivas (Lachkhem et al., 2008; Khelifi et al., 2009; Haldar et al.; Artigas Raventós et al.; Song et al., 2017). Los resultados de protocolos de quimio y/o radioterapia so pobres dada la gran cantidad de colágeno en este tipo neoplasias; lo que se asocia a la escasa celularidad y baja actividad mitótica que los caracteriza (Haldar et al.; Song et al.). No obstante ello, existe cierta evidencia, de posible respuesta terapéutica a tratamientos hormonales, en aquellos casos de recpetores estrogénicos positivos (Rotmensch et al.).

Por su parte, la endometriosis infiltrativa profunda (EIP), se trata de una patología que aunque hace parte del síndrome endometriósico, es claramente diferente de la endometriosis superficial (De Los Ríos-Posada, 2012). Se ha postulado una etiopagenia distinta de la endometriosis (teoría de Sampson); y se caracterizaría por remanentes müllerianos que sufren metaplasia, convirtiéndose en fibroblastos, células musculares lisas y componentes glandulares y del estroma endometrial (Donnez et al., 1995).

\footnotetext{
${ }^{1}$ Departamento de Cirugía y Centro de Excelencia en Estudios Morfológicos y Quirúrgicos (CEMyQ), Universidad de La Frontera, Chile.

${ }^{2}$ Clínica RedSalud Mayor Temuco, Chile.
} 
También se han reportado diferencias histológicas entre endometriosis y EIP; caracterizándose esta última por la existencia de células de músculo liso y reacción fibrosa, alternadas células endometriales escasas. A esto, se suma eventualmente la presencia de células glandulares sumidas en una matriz muscular; hallazgos que ha hecho plantear que esta entidad se trata más bien de una adenomiosis (Signorile et al., 2009). Asimismo existe evidencia de diferencias inmunológicas con la endometriosis común; destacando las mediciones de interleukinas 2, 4 y 10, de factor de necrosis tumoral alfa, e interferón gamma (Podgaec et al., 2010).

Presenta un comportamiento semejante a una neoplasia, con capacidad de invasión locoregional, linfangiogénesis y desarrollo de metástasis vía linfática, encontrándose células endometriósicas en linfonodos de la pelvis (Noel et al., 2008; Bassi et al., 2009; Keichel et al., 2011). De este modo, suele afectar el compartimiento anterior (infiltración de ligamentos redondos y de la pared vesical) (Crispi et al., 2012); la pared lateral de la pelvis (infiltración de uréteres, plexos nerviosos hipogástricos, etc.) (Chapron et al., 2010; Ceccaroni et al., 2011; Muñoz et al., 2012); el compartimiento posterior (compromiso de parametrios, ligamentos uterosacros, vagina, el tabique rectovaginal y el recto) (Chopin et al., 2005; Dadhwal et al., 2012; Milone et al., 2015); y otro órganos como intestino delgado, sigmoides, ciego y apéndice cecal (Fernández-Rey et al., 2009; Villarreal-Peral et al., 2011). Esto, significa que su tratamiento de la EIP es la ablación quirúrgica es de una radicalidad que implica la necesidad de accesos retroperitoneales extensos, exéresis y reparación de órganos comprometidos, procedimientos muy distintos de la cirugía de la endometriosis peritoneal (Vercellini et al., 2009; De Los Ríos-Posada; Muñoz et al.).

\section{CASO CLÍNICO}

Se trata de una paciente de sexo femenino, 41 años; que consultó por disuria, tenesmo rectal y dolor pélvico. Había sido intervenida recientemente en otro centro por endometriosis.

La exploración física permitió detectar la protrusión de una lesión tumoral que protruía por la vagina de la paciente; la que fue biopsiada y cuyo informe fue de angiomixoma.

Se solicitó una colonoscopia, que permitió verificar la presencia de una masa tumoral móvil, localizada a $12 \mathrm{~cm}$ por encima del margen anal; que ocupaba la casi totalidad de la luz rectal, de consistencia pétrea y aspecto neoproliferativo (Fig. 1), razón por la que se tomaron muestras para biopsia.

La tomografía computarizada, permitió verificar la existencia de una imagen tumoral de $15 \mathrm{~cm}$, localizada por detrás de la vejiga, fusionada al recto medio; con burbujas aéreas en su interior (Fig. 2). Además, de compromiso de la cúpula vaginal, cuello uterino y anexos. Simultáneamente se constató gran hidro-uretero-nefrosis izquierda.

Fue intervenida quirúrgicamente el 13 de diciembre de 2018; oportunidad en la que se realizó rectosigmoidectomía a lo Hartmann (Fig. 3), nefrectomía izquierda y exéresis en bloque del útero, anexos y pared vaginal superior (Fig. 4), y de linfonodos regionales.

La paciente evolucionó de forma favorable, sin complicaciones y fue dada de alta al $5^{\circ}$ día del postoperatorio en buenas condiciones.
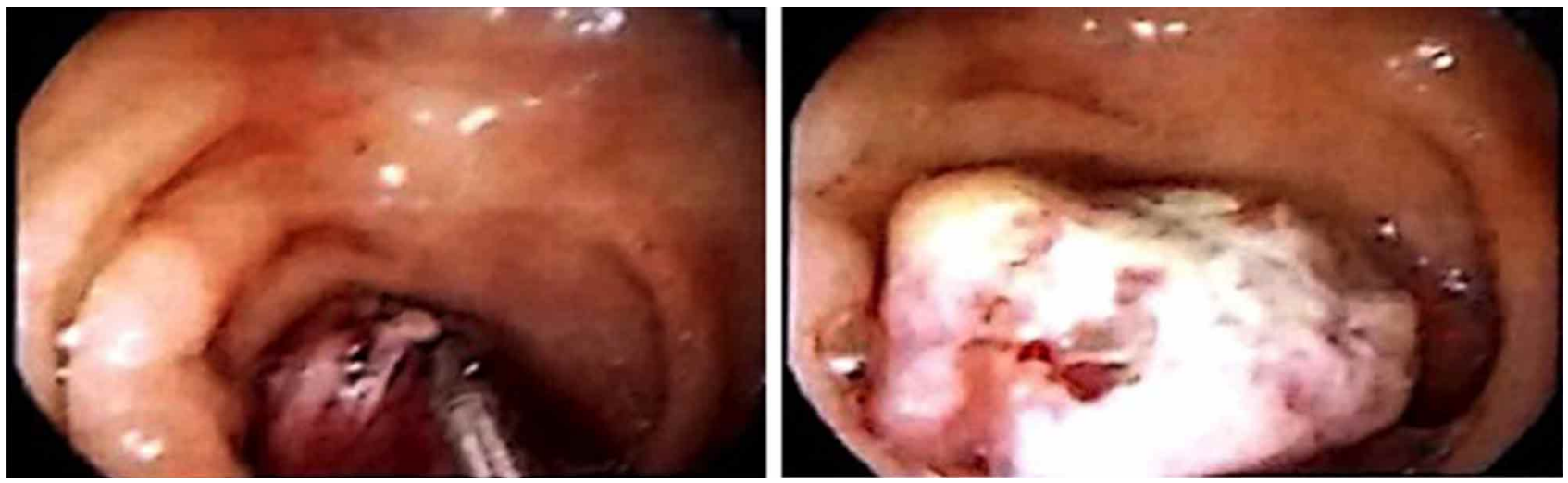

Fig. 1. Colonoscopia. Se aprecia la existencia de una masa tumoral, que ocupaba la casi totalidad de la circunferencia rectal, de aspecto neoproliferativo. 


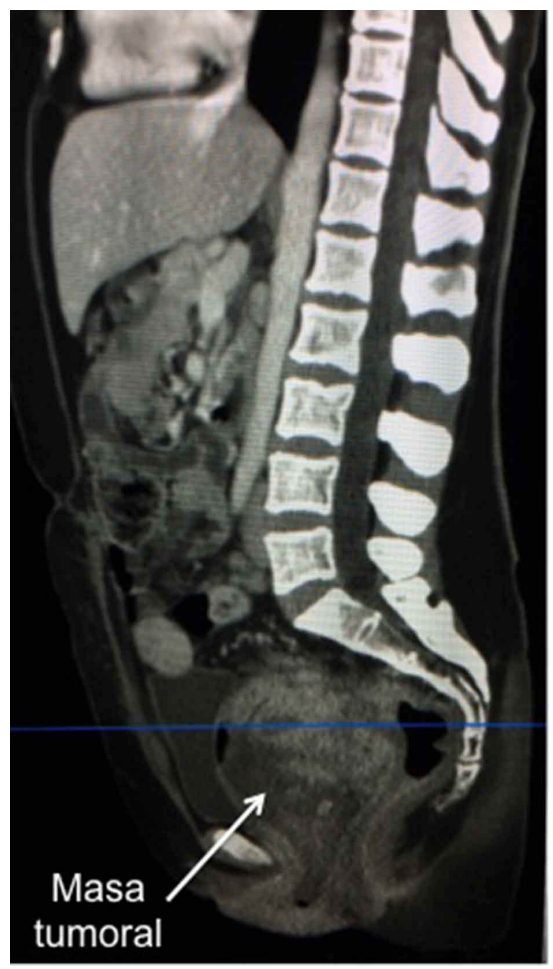

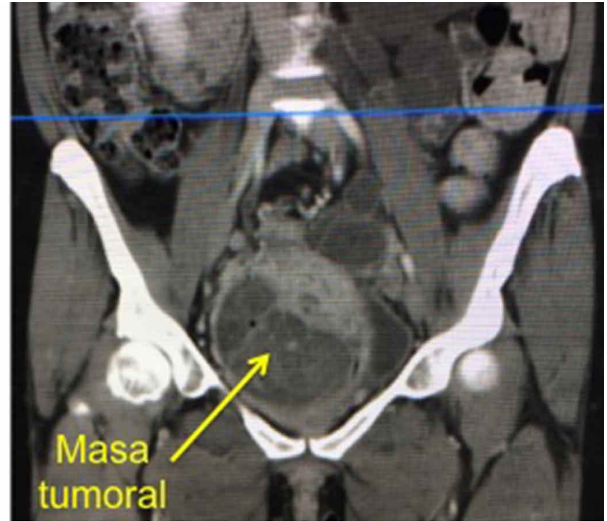

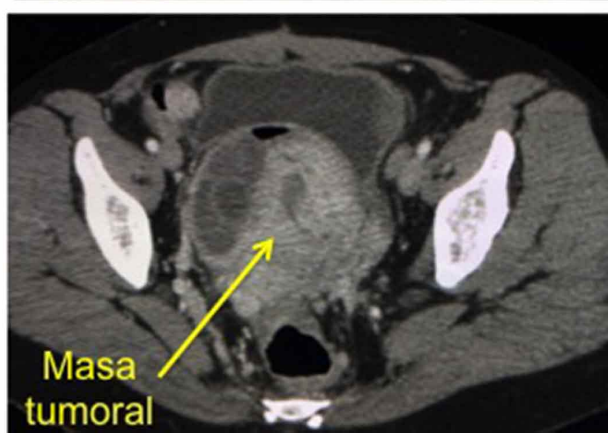

Fig. 2. Imágenes axial, sagital y coronal de tomografía computarizada; en la que se aprecia una imagen tumoral de $15 \mathrm{~cm}$, localizada por detrás de la vejiga, fusionada al recto medio; en cuyo interior destaca una burbuja aérea.
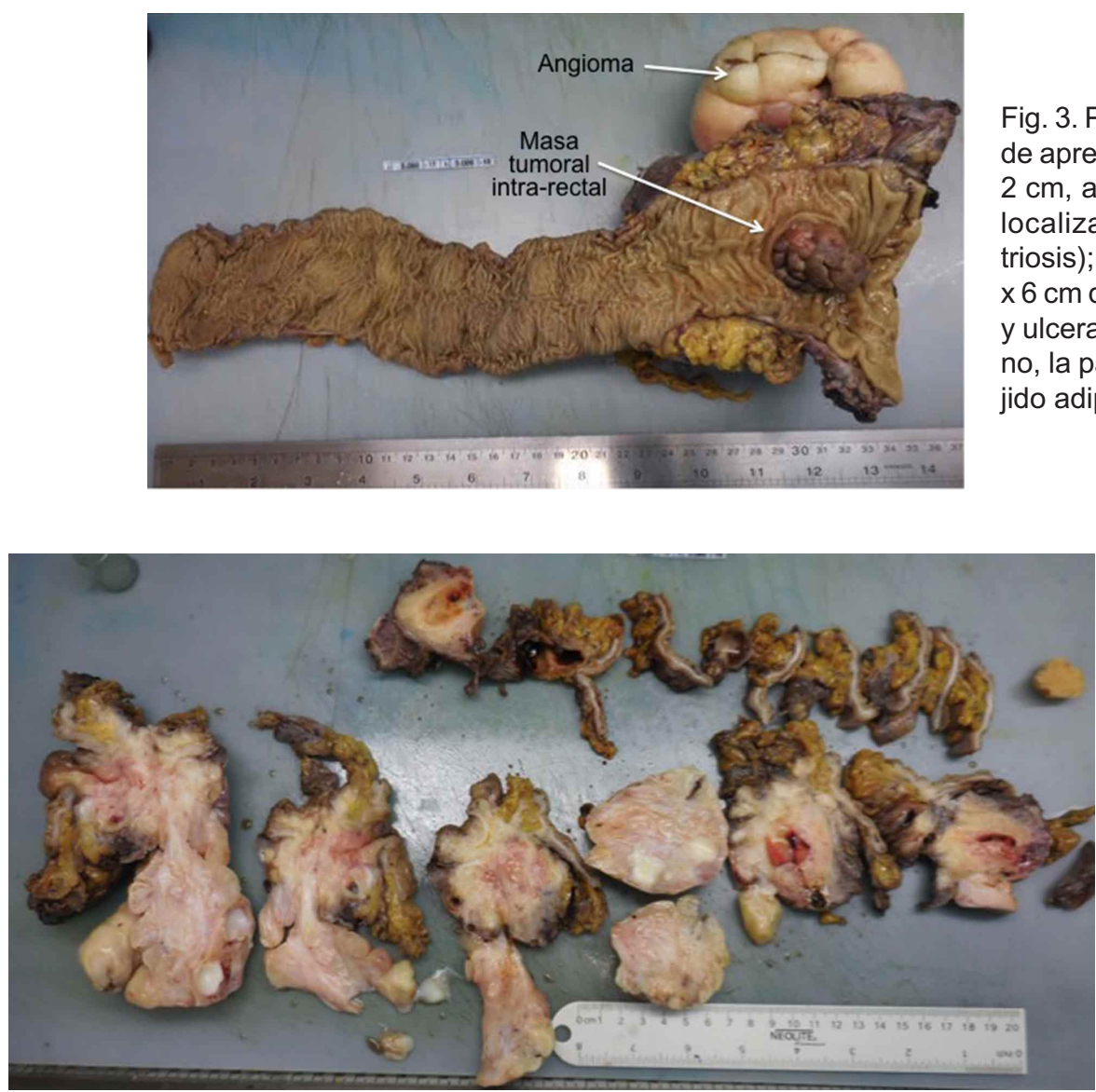

Fig. 3. Pieza de colectomía izquierda. Se puede apreciar una lesión polipoidea sésil, de $4 \mathrm{x}$ $2 \mathrm{~cm}$, a $5 \mathrm{~cm}$ del margen de sección caudal, localizada en recto medio-bajo (endometriosis); así como un angiomixoma, de $10 \times 8$ $x 6 \mathrm{~cm}$ de ejes mayores, con áreas necróticas y ulceradas, que comprometía el cuello uterino, la pared vaginal, infiltrando además el tejido adiposo circundante y la pared rectal.
Fig. 4. Pieza quirúrgica. Se observan diversos cortes del angiomixoma que comprometía cuello uterino, pared vaginal, pared rectal y tejido adiposo circundante. 

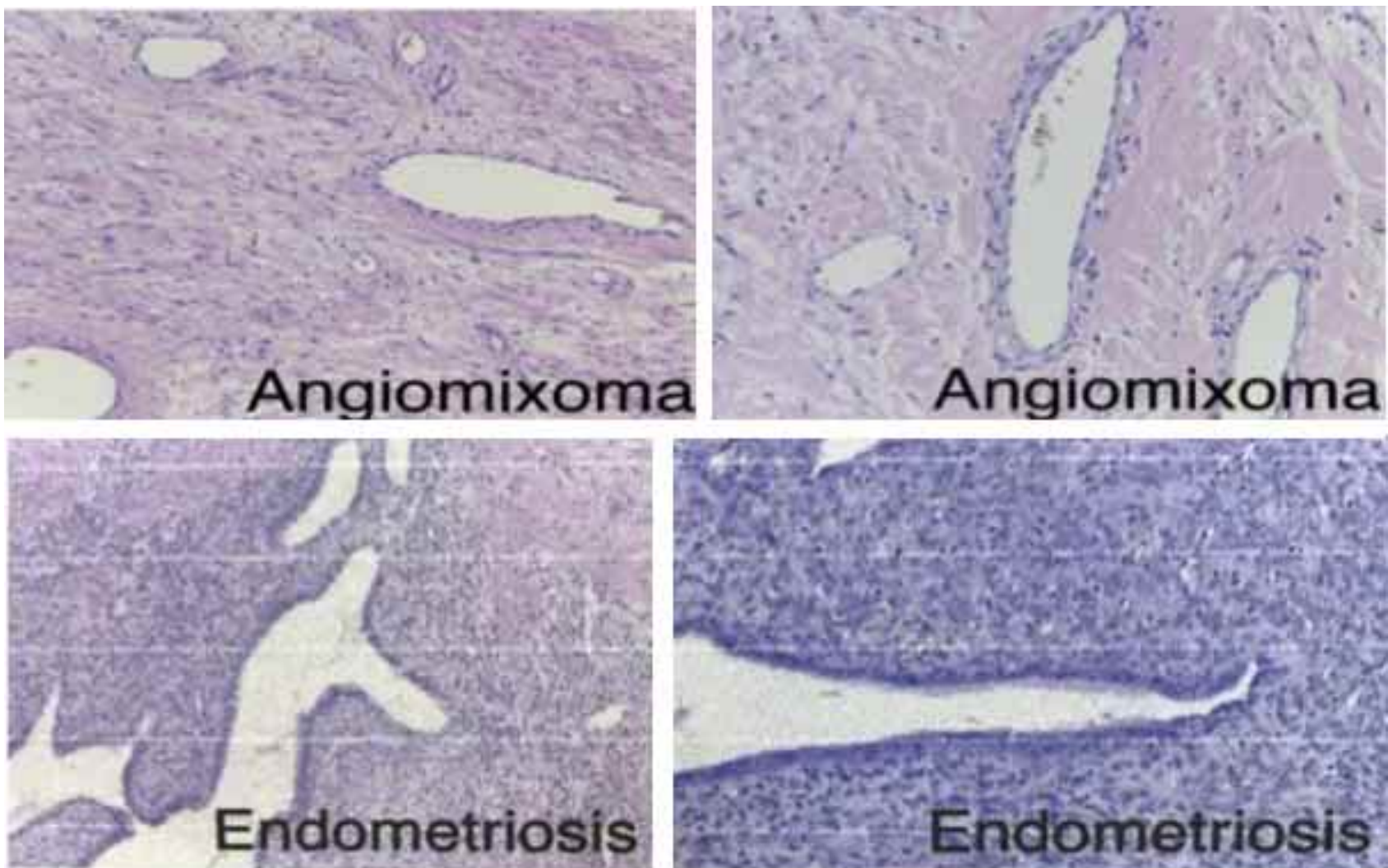

Fig. 5. Microfotografías. Se aprecian cortes histológicos con imágenes del angiomixoma y de un foco de endometriosis infiltrativa profunda.

Informe de histopatología fue angiomixoma de tipo fibromixoide, con vasos sanguíneos de tamaño variable, y sin áreas de necrosis ni hemorragia, con compromiso de pared vaginal y cuello uterino; y endometriosis infiltrativa profunda en pared de recto, zona pericervical, de pared vaginal y tejido adiposo periureteral izquierdo (Fig. 5). No se encontró evidencias de infiltración linfonodal (0 de 5 linfonodos examinados), ni neoplásica en riñón y uréter izquierdo, y colon sigmoides. La inmunohistoquímica fue positiva para receptores de estrógeno y progesterona Mouse Monoclonal 6F11 y 16 respectivamente.

Al control postoperatorio del tercer mes, la pacientes se encuentra asintomática, con alza de peso, utilizando de forma adecuada su colostomía. La tomografía de control, no muestra imágenes de recidiva.

\section{CONCLUSION}

Podemos señalar que el angiomixoma de la pelvis tumor mesenquimal inusual; que dado su alto potencial de recidiva es relevante que la exéresis quirúrgica sea lo más radical posible. Por su parte, la EIP es otra entidad poco frecuente que requiere de tratamientos complejos. La coexistencia de ambas patologías representa una situación absolutamente infrecuente; de los que hay muy pocos casos reportado en la literatura occidental (Coyne, 2012).

MANTEROLA, C.; SAN MARTÍN, R. \& CHODOWIECKI, A. Pelvic Angiomyxoma associated to deep infiltrative endometriosis. Case report. Int. J. Morphol., 37(2):671-676, 2019.

SUMMARY: Angiomyxoma of the pelvis is an unusual mesenchymal tumor; that given its high potential for recurrence, it is relevant that the surgical resection be as radical as possible. For its part, to deep infiltrative endometriosis is another rare entity that requires complex treatments. The co-existence of both pathologies represents an absolutely infrequent situation; of which there are very few cases reported in western literature. We present the case of a 41-year-old woman who developed both clinical entities concomitantly and who was treated surgically with good results.

KEY WORD: Angiomyxoma; Myxoma; Neoplasms, Connective Tissue; Pelvic Neoplasms. 


\section{REFERENCIAS BIBLIOGRÁFICAS}

Adwan, H.; Patel, B.; Kamel, D. \& Glazer, G. A solitary encapsulated pelvic aggressive angiomyxoma. Ann. R. Coll. Surg. Engl., 86(6):W1-3, 2004.

Andres, L. A. \& Mishra, M. J. Angiomyxoma diagnosed in a man presenting for abdominal lipectomy. Can. J. Plast. Surg., 15(3):163-4, 2007.

Artigas Raventós, V.; Rivero Cruces, D.; González López, J.; Rodríguez Blanco, M. \& Boguña, I. Aggressive angiomyxoma. A rare mesenchymal pelvic tumor. Cir. Esp., 94(6):359-61, 2016.

Bassi, M. A.; Podgaec, S.; Dias Júnior, J. A.; Sobrado, C. W. \& D Amico Filho, N. Bowel endometriosis: a benign disease? Rev. Assoc. Med. Bras. (1992), 55(5):611-6, 2009.

Ceccaroni, M.; Clarizia, R.; Cosma, S.; Pesci, A.; Pontrelli, G. \& Minelli, L. Cyclic sciatica in a patient with deep monolateral endometriosis infiltrating the right sciatic nerve. J. Spinal Disord. Tech., 24(7):474-8, 2011.

Chapron, C.; Chiodo, I.; Leconte, M.; Amsellem-Ouazana, D.; Chopin, N.; Borghese, B. \& Dousset, B. Severe ureteral endometriosis: the intrinsic type is not so rare after complete surgical exeresis of deep endometriotic lesions. Fertil. Steril., 93:2115-20, 2010.

Chen, H.; Zhao, H.; Xie, Y. \& Jin, M. Clinicopathological features and differential diagnosis of aggressive angiomyxoma of the female pelvis: 5 case reports and literature review. Medicine (Baltimore), 96(20):e6820, 2017.

Choi, H.; Park, C. \& Ji, Y. I. Alternative surgical approaches for aggressive angiomyxoma at different sites in the pelvic cavity. Obstet. Gynecol. Sci., 58(6):525-9, 2015.

Chopin, N.; Vieira, M.; Borghese, B.; Foulot, H.; Dousset, B.; Coste, J.; Mignon, A.; Fauconnier, A. \& Chapron, C. Operative management of deeply infiltrating endometriosis: results on pelvic pain symptoms according to a surgical classification. J. Minim. Invasive Gynecol., 12(2):106-12, 2005.

Coyne, J. D. Aggressive angiomyxoma admixed with endometriosis: a case report. Int. J. Surg. Pathol., 20(2):205-7, 2012.

Crispi, C. P.; de Souza, C. A.; Oliveira, M. A.; Dibi, R. P.; Cardeman, L.; Sato, H. \& Schor, E. Endometriosis of the round ligament of the uterus. J. Minim. Invasive Gynecol., 19(1):46-51, 2012.

Dadhwal, V.; Deka, D.; Mathur, S.; Kaushal, S.; Sharma, A. K. \& Mittal, S. Vaginal polypoid endometriosis simulating neoplasia in a young woman. J. Low Genit. Tract Dis., 16(3):318-21, 2012.

De Los Ríos-Posada, J. F. ¿Por qué la endometriosis infiltrativa profunda debe ser considerada una entidad diferente a la endometriosis peritoneal? Rev. Colomb. Obstet. Ginecol., 63(3):259-71, 2012.

Donnez, J.; Nisolle, M.; Casanas-Roux, F.; Bassil, S. \& Anaf, V. Rectovaginal septum, endometriosis or adenomyosis: laparoscopic management in a series of 231 patients. Hum. Reprod., 10(3):630-5, 1995.

Fernández-Rey, C. L.; Alvarez-González, S. A.; Díaz-Solís, P.; BlancoGonzález, A. \& Costilla-García, S. Small bowel obstruction secondary to ileal endometriosis: multisection computer tomography evaluation. Rev. Esp. Enferm. Dig., 101(12):872-4, 2009.

Giraudmaillet, T.; Mokrane, F. Z.; Delchier-Bellec, M. C.; Motton, S.; Cron, C. \& Rousseau, H. Aggressive angiomyxoma of the pelvis with inferior vena cava involvement: MR imaging features. Diagn. Interv. Imaging, 96(1):111-4, 2015.

Haldar, K.; Martinek, I. E. \& Kehoe, S. Aggressive angiomyxoma: a case series and literature review. Eur. J. Surg. Oncol., 36(4):335-9, 2010.

Idrees, M. T.; Hoch, B. L.; Wang, B. Y. \& Unger, P. D. Aggressive angiomyxoma of male genital region. Report of 4 cases with immunohistochemical evaluation including hormone receptor status. Ann. Diagn. Pathol., 10(4):197-204, 2006.

Keichel, S.; Barcena de Arellano, M. L.; Reichelt, U.; Riedlinger, W. F.; Schneider, A.; Köhler, C. \& Mechsner, S. Lymphangiogenesis in deep infiltrating endometriosis. Hum. Reprod., 26(10):2713-20, 2011.
Khelifi, S.; Ben Ali, A.; Tagougui, W.; Jaoua, H.; Chammakhi, C.; Chadly, A.; Bouhafa, A.; Maamar, A. \& Cherif, A. Perineal recurrence of an aggressive angiomyxoma: Is an incomplete resection useful? J. Chir. (Paris), 146(4):416-8, 2009.

Kiran, G.; Yancar, S.; Sayar, H.; Kiran, H.; Coskun, A. \& Arikan, D. C. Late recurrence of aggressive angiomyxoma of the vulva. J. Low Genit. Tract Dis., 17(1):85-7, 2013.

Lachkhem, A.; Mardassi, S.; Ben Gamra, S.; Ouertani, H.; Kochbati, L.; Elmay, A.; Touati, S. \& Gritli, S. Angiomyxome agressif de la joue : a propos d'une observation. J. Tun. ORL, (20):59-61, 2008.

Linares Espinos, E.; Rengifo Abbad, D.; Van de Brule Rodriguez de Medina, E.; Osorio Cabello, L.; Areche Espiritusanto, J. \& Carballido Rodriguez, J. Aggressive pelvic angiomyxoma. Arch. Esp. Urol., 67(3):288-90, 2014.

Llauger, J.; Palmer, J.; Pérez, C.; Monil, J.; Ribé, J. \& Moreno, A. The normal and pathologic ischiorectal fossa at CT and MR imaging. Radiographics, 18(1):61-82, 1998.

Milone, M.; Vignali, A.; Milone, F.; Pignata, G.; Elmore, U.; Musella, M.; De Placido, G.; Mollo, A.; Fernandez, L. M.; Coretti, G.; et al. Colorectal resection in deep pelvic endometriosis: Surgical technique and postoperative complications. World J. Gastroenterol., 21(47):13345-51, 2015.

Muñoz, J. L.; Jiménez, J. S.; Tejerizo, A.; Lopez, G.; Duarte, J. \& Sánchez Bustos, F. Rectosigmoid deep infiltrating endometriosis and ureteral involvement with loss of renal function. Eur. J. Obstet. Gynecol. Reprod. Biol., 162(2):121-4, 2012.

Noel, J. C.; Chapron, C.; Fayt, I. \& Anaf, V. Lymph node involvement and lymphovascular invasion in deep infiltrating rectosigmoid endometriosis. Fertil. Steril., 89(5):1069-72, 2008.

Podgaec, S.; Dias Junior, J. A.; Chapron, C.; Oliveira, R. M.; Bacarat, E. C. \& Abrão, M. S. Th1 and Th2 ummune responses related to pelvic endometriosis. Rev. Assoc. Med. Bras. (1992), 56(1):92-8, 2010.

Rotmensch, E. J.; Kasznica, J. \& Hamid, M. A. Immunohistochemical analysis of hormone receptors and proliferating cell nuclear antigen in aggressive angiomyxoma of the vulva. Int. J. Gynaecol. Obstet., 41(2):171-9, 1993.

Signorile, P. G.; Campioni, M.; Vincenzi, B.; D`Avino, A. \& Baldi, A. Rectovaginal septum endometriosis: an immunohistochemical analysis of 62 cases. In Vivo, 23(3):459-64, 2009.

Song, M.; Glasgow, M.; Murugan, P. \& Rivard, C. Aggressive angiomyxoma of the vulva and bladder. Obstet. Gynecol., 130(4):885-8, 2017.

Steeper, T. A. \& Rosai, J. Aggressive angiomyxoma of the female pelvis and perineum. Report of nine cases of a distinctive type of gynecologic soft-tissue neoplasm. Am. J. Surg. Pathol., 7(5):463-75, 1983.

Sutton, B. J. \& Laudadio, J. Aggressive angiomyxoma. Arch. Pathol. Lab. Med., 136(2):217-21, 2012.

Vercellini, P.; Carmignani, L.; Rubino, T.; Barbara, G.; Abbiati, A. \& Fedele, L. Surgery for deep endometriosis: a pathogenesis-oriented approach. Gynecol. Obstet. Invest., 68(2):88-103, 2009.

Villarreal-Peral, C.; Olvera-Gracida, L.; González-Maynes, M. de L. \& Saucedo-Ruiz, G. Appendicular endometriosis as a cause of acute abdomen. Ginecol. Obstet. Mex., 79(8):489-92, 2011.

Dirección para correspondencia:

Dr. Carlos Manterola. Departamento de Cirugía y CEMyQ

Universidad de La Frontera

Manuel Montt 112, oficina 408

Temuco - CHILE

\section{E-mail: carlos.manterola@ufrontera.cl}

Recibido : 11-11-2018

Aceptado: 16-02-2019 